\title{
BMP2-dependent gene regulatory network analysis reveals Klf4 as a novel transcription factor of osteoblast differentiation
}

Shuaitong Yu', Jinqiang Guo', Zheyi Sun ${ }^{1}$, Chujiao Lin ${ }^{1}$, Huangheng Tao', Qian Zhang ${ }^{1}$, Yu Cui ${ }^{1}$, Huanyan Zuo', Yuxiu Lin ${ }^{1}$, Shuo Chen', Huan Liu (10) ${ }^{1,3}$ and Zhi Chen (iD)

\begin{abstract}
Transcription factors (TFs) regulate the expression of target genes, inducing changes in cell morphology or activities needed for cell fate determination and differentiation. The BMP signaling pathway is widely regarded as one of the most important pathways in vertebrate skeletal biology, of which BMP2 is a potent inducer, governing the osteoblast differentiation of bone marrow stromal cells (BMSCs). However, the mechanism by which BMP2 initiates its downstream transcription factor cascade and determines the direction of differentiation remains largely unknown. In this study, we used RNA-seq, ATAC-seq, and animal models to characterize the BMP2-dependent gene regulatory network governing osteoblast lineage commitment. Sp7-Cre; Bmp $2^{\mathrm{fx} / \mathrm{fx}}$ mice (BMP2-cKO) were generated and exhibited decreased bone density and lower osteoblast number $(n>6)$. In vitro experiments showed that BMP2-cKO mouse bone marrow stromal cells (mBMSCs) had an impact on osteoblast differentiation and deficient cell proliferation. Osteogenic medium induced mBMSCs from BMP2-cKO mice and control were subjected to RNA-seq and ATAC-seq analysis to reveal differentially expressed TFs, along with their target open chromatin regions. Combined with H3K27Ac CUT\&Tag during osteoblast differentiation, we identified 2338 BMP2-dependent osteoblast-specific active enhancers. Motif enrichment assay revealed that over $80 \%$ of these elements were directly targeted by RUNX2, DLX5, MEF2C, OASIS, and KLF4. We deactivated KIf4 in the Sp7 + lineage to validate the role of KLF4 in osteoblast differentiation of mBMSCs. Compared to the wild-type, Sp7-Cre; KIff ${ }^{f \times /+}$ mice (KLF4-Het) were smaller in size and had abnormal incisors resembling BMP2-cKO mice. Additionally, KLF4-Het mice had fewer osteoblasts and decreased osteogenic ability. RNA-seq and ATAC-seq revealed that KLF4 mainly "co-bound" with RUNX2 to regulate downstream genes. Given the significant overlap between KLF4- and BMP2-dependent NFRs and enriched motifs, our findings outline a comprehensive BMP2-dependent gene regulatory network specifically governing osteoblast differentiation of the Sp7 + lineage, in which Klf4 is a novel transcription factor.
\end{abstract}

Correspondence: Huan Liu (liu.huan@whu.edu.cn) or

Zhi Chen (zhichen@whu.edu.cn)

${ }^{1}$ State Key Laboratory Breeding Base of Basic Science of Stomatology (HubeiMOST) and Key Laboratory for Oral Biomedicine of Ministry of Education (KLOBM), School and Hospital of Stomatology, Wuhan University, Wuhan,

China

${ }^{2}$ Department of Developmental Dentistry, University of Texas Health Science Center, San Antonio, TX, USA

Full list of author information is available at the end of the article

Edited by A. Stephanou

\section{Introduction}

The vertebrate skeleton is made of cartilage and bone, which is derived from three different embryonic lineages $^{1}$. Cells arising in these lineages proliferate and migrate to form mesenchymal condensations ${ }^{2}$. Embryonic mesenchymal cells differentiate into chondrocytes in cartilage, osteoblasts, and osteoclasts in bone. Intramembranous ossification and endochondral ossification are two major methods of mesenchymal cells involved in bone formation ${ }^{3,4}$. In both approaches,

\section{(c) The Author(s) 2021}

(c) Open Access This article is licensed under a Creative Commons Attribution 4.0 International License, which permits use, sharing, adaptation, distribution and reproduction cc) in any medium or format, as long as you give appropriate credit to the original author(s) and the source, provide a link to the Creative Commons license, and indicate if changes were made. The images or other third party material in this article are included in the article's Creative Commons license, unless indicated otherwise in a credit line to the material. If material is not included in the article's Creative Commons license and your intended use is not permitted by statutory regulation or exceeds the permitted use, you will need to obtain permission directly from the copyright holder. To view a copy of this license, visit http://creativecommons.org/licenses/by/4.0/. 
mesenchyme-derived osteoblasts play a critical role in bone formation and, ultimately, bone remodeling, involving a complex gene regulatory network. Gene expression is orchestrated by cis-regulated elements, including enhancers and promoters, that recruit transcription factors and their cofactors ${ }^{5}$. Transcription factors (TFs) can bind to specific DNA motifs and lead to an on/off mode of transcriptional regulation, which eventually determines the spatial and temporal expression of genes. TFs have been reported to be key factors in stem cell pluripotency maintenance and cell fate decision ${ }^{6,7}$. A clear understanding of how tissue-specific TFs interact with each other and their downstream targets will provide insights into the mechanism of gene regulation in osteoblast differentiation.

Bone morphogenetic proteins (BMPs), members of the transforming growth factor beta (TGF- $\beta$ ) superfamily, regulate skeletal development and tissue homeostasis ${ }^{8,9}$ through a cascade of downstream signal transduction. As multifunctional proteins, BMPs are considered one of the key mediators in vertebrate skeletal biology. Among them, bone morphogenetic protein 2 (BMP2), a pivotal family member, has been abundantly reported as a major inducer of cartilage and bone formation ${ }^{10-13}$. The potent osteogenic effects of BMP2 are mediated in part by the formation of RUNX2-SMAD complexes ${ }^{14,15}$. In addition, BMP2 can induce SP7(Osterix) expression by $D l x 5^{16}$ and induce ALPL expression in a Wnt autocrine loop ${ }^{17}$ in vitro. Using a conditional knockout mouse model, the function of BMP2 in different lineages has also been studied. Sp7-Cre mouse lines characterize early osteoblast precursors, making it easier to observe osteoblast development ${ }^{18}$. Previous studies using $S p 7-\mathrm{Cre} ; \mathrm{Bmp} 2^{\mathrm{fx} / \mathrm{fx}}$ mice demonstrated that BMP2 can determine the specification of osteoblast cell fate ${ }^{19}$, long bone strength ${ }^{20}$, and endochondral healing ${ }^{21}$. It can also regulate enamel-related gene expression ${ }^{22,23}$ and control tooth root development $^{24}$. These studies verified the significance of BMP2 in mineralized tissue development in vivo. However, the detailed regulatory mechanism of how BMP2 initiates its downstream transcription factors to directly determine or affect mesenchymal cell differentiation remain largely unclear.

In this study, we attempted to determine the downstream functional TFs by analyzing the enrichment of their fingerprint through changes in open chromatin regions, which are associated with cell fate decision ${ }^{25-27}$. Hence, we inactivated $B m p 2$ in early osteoblast precursors using $S p 7-\mathrm{Cre}$ in vivo and then utilized RNA-seq and ATAC-seq to profile the target of BMP2 during osteoblast differentiation in vitro. In summary, the present study outlines a comprehensive BMP2-dependent gene regulatory network governing osteoblast differentiation of the $S p 7+$ lineage.

\section{Results}

Loss of Bmp2 in Sp7+ lineage inhibits postnatal bone development by inhibiting osteoblastogenesis

The Sp7-Cre; $B m p 2^{\mathrm{fx} / \mathrm{fx}}$ mice (BMP2-cKO) were fertile, and newborns appeared normal. Skeletal staining at PN 0.5 suggested that there were no significant differences between the skeletons of the mutants and their wild-type siblings (Fig. 1A and S1B-E). The forelimbs (Fig. S1F) and hindlimbs (Fig. 1B) were normal in length but slightly thinner and underdeveloped.

However, as early as 2 weeks after birth, these mutant mice were smaller in size. At 8 weeks after birth, BMP2cKO mice displayed a visibly smaller body size (Fig. 1C). The X-ray and micro-CT results showed underdeveloped long bones and decreased bone density in the mutant (Fig. 1D-F). H\&E staining confirmed that the trabecular bone number and the width of cortical bone were reduced in BMP2-cKO (Fig. 1G). At around 16 weeks after birth, the BMP2-cKO mice exhibited abnormal incisors and mandibles (Fig. S1G-J). These craniofacial phenotypes were in agreement with a previous study ${ }^{23}$.

The detailed histological changes in BMP2-cKO mice of different ages were same like 8 weeks' mutant mice (Fig. S2A-C). Safranin O staining showed irregularly arranged and abnormally shaped chondrocytes in the mutant (Fig. S2D).

We then asked if such defects in bone development were due to either cellular proliferation or osteoblast differentiation. In 8-week-old mice, Ki67 staining revealed reduced cellular proliferation near the growth plate and bone trabeculae. We then performed immunohistochemistry for osteogenic-related genes, SP7 and OPN, markers for preosteoblasts and differentiated osteoblasts, respectively ${ }^{28}$. Near the epiphysis and metaphysis of femurs, the SP7-positive and OPN-positive domains were significantly reduced in the mutant (Fig. S3A). These results indicated that $B m p 2$ deficiency in the $S p 7+$ lineage resulted in a widespread reduction of cellular proliferation, which impacted osteoblast differentiation in vivo.

To confirm whether the deletion of Bmp2 in the Sp7+ lineage inhibits osteoblast differentiation in vitro, we collected mBMSCs which were maintained in the osteogenic medium. Loss of Bmp2 in BMP2-cKO cells was confirmed (Fig. S4D). In the Bmp2-WT groups, $B m p 2$ was found to increase dramatically, while in the BMP2-cKO group it remained at a low level, which may be due to deletion of $B m p 2$ upon osteogenic induction. We further examined the expression of osteogenesis-related genes by western blotting and qPCR. These maker genes remained at a significantly lower level in the BMP2-cKO group compared with the control (Fig. S3C-G). Alizarin red staining showed the number of calcium nodules was decreased in BMP2-cKO (Fig. S3B). Interestingly, we found that the loss of $B m p 2$ also affected the ability of 


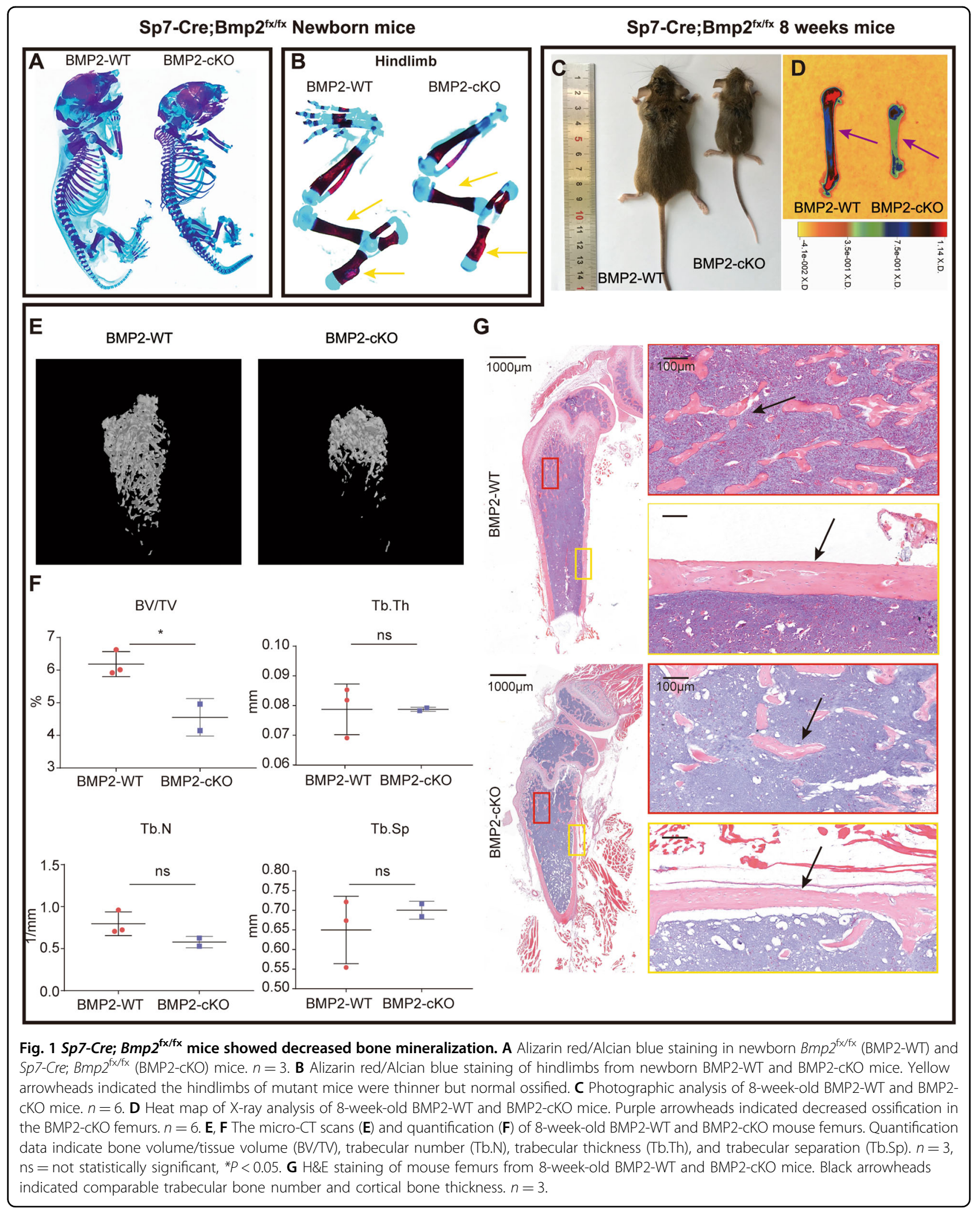


mBMSCs to proliferate (Fig. S4A-C) and undergo adipogenesis (Fig. S4E-G). In summary, the inactivation of $B m p 2$ in the $S p 7+$ lineage impedes postnatal osteogenesis and inhibits cell proliferation, osteoblastogenesis, and adipogenesis in mBMSCs.

\section{Using RNA-seq and ATAC-seq reveals BMP2-denpendent gene regulatory network}

To identify all potential transcription factors regulated by BMP2 in the $S p 7+$ lineage and comment on the main BMP2-related pathway(s) and transcriptional mechanism (s) during osteoblast differentiation, we utilized RNA-seq and ATAC-seq (Fig. 2A).

Based on RNA-seq results, we identified 378 genes with a significant change $(P<0.01$ and $q<0.05)$ of expression levels due to $B m p 2$ deficiency. Among them, 142 genes were significantly downregulated by $B m p 2$ deficiency $(\beta$-value $<0)$, and 236 genes were upregulated. The downregulated genes were significantly enriched for mineralization-related GO terms, such as bone mineralization (GO: 0030282) and osteoblast differentiation (GO: 0001649; Fig. 2C), including Wnt10, Klf4, Mef2d, Atf4, and Smad6 (Fig. 2B).

Next, we identified nucleosome-free regions (NFRs) from the ATAC-seq results described above, mapping them within the mouse genome. Regions of both lost- and gained-genome accessibility were observed with $B m p 2$ deficiency (Fig. 2D, E). Overall, the ATAC-seq signals were enriched in putative distal regulatory regions, suggesting that abundant enhancers are involved in open chromatin accessibility (Fig. S5A, B). To identify the biological process and mouse phenotype enrichment for the nearby genes of Bmp2 deficiency lost- and gainedNFRs, we used the GREAT algorithm. We found that Bmp2 deficiency resulted in the loss of NFRs enriched in bone and chondrocyte development (Fig. S5C), and the relevant mice exhibited both osteoblast and osteoclast deficiencies (Fig. 2F). While Bmp2 deficiency resulted in the gain of NFRs enriched in various biological process (Fig. S5D). In summary, by combining RNA-seq with ATAC-seq, we found that the loss of BMP2 in $S p 7+$ lineage BMSCs resulted in a decrease in the expression and accessibility of nucleosome-free regions near osteogenesis-related genes, which is responsible for the bone defects observed in BMP2-cKO.

\section{KIf4 is a BMP2-dependent potential transcription factor governing osteoblast differentiation}

Since nucleosome-free regions that are directly targeted by transcription factors are associated with different biological processes ${ }^{29}$, we investigated whether we could infer any functional TFs by analyzing the enriched motifs within the BMP2-dependent NFRs. Using HOMER, we identified the five most enriched DNA sequence motifs, corresponding to the preferred binding sites of specific transcription factors, to identify the major functional TFs of Bmp2 in the Sp7+ lineage. Overall, over $80 \%$ of these cis-regulatory elements were directly targeted by these five significantly enriched motifs (Fig. 2G). In addition, we corresponded these enriched motifs with bestmatched TFs according to their expression level, identified by RNA-seq. Among them, the DLX and RUNX family members ranked at the top, among which $D l x 5$ and Runx2 are critical for osteoblast differentiation ${ }^{30-33}$. We also observed the enrichment of the MADS and bZIP families, whose best match transcription factors, $M e f 2 c^{34}$ and $\mathrm{Oasis}^{35}$, were expected to be meaningful TFs in osteoblast differentiation. However, BMP2-cKO gained NFRs showed different motif enrichment (Fig. S5E). Next, we used Cytoscape to build a network linking the major BMP2-WT enriched motifs and the best-matched candidate transcription factors. This network model supported the hypothesis that BMP2 regulates downstream transcription factors mainly through the Smad-dependent pathway (Fig. S6). Interestingly, we noticed that KLF motifs were highly enriched in lost NFRs. Integrated with our RNA-seq results simultaneously, we focused on a member of the KLF motif family Klf4, which has rarely been reported in osteoblast differentiation.

To annotate the active enhancers/promoters among the BMP2-dependent open chromatin regions, we integrated the ATAC-seq results with the H3K27Ac CUT\&Tag results from the MC3TC-E1 cell line ${ }^{36}$. The integration of H3K27Ac and ATAC-seq results revealed that 2338 active enhancers were BMP2-dependent in the $S p 7+$ lineage. In the "gene desert" downstream of the Klf4 locus, we noticed several ATAC-seq peaks were "closed" in the BMP2-cKO group (Fig. 3A), indicating that BMP2 regulates Klf4 transcription by regulating these osteoblast-associated enhancers. To evaluate whether these NFRs exert enhancer activity for Klf4, we chose two representative NFRs. Both were upstream of Klf4: K2 (mm10: chr4:5563629055636742) and K6 (mm10: chr4:56136777-56137230). The dual luciferase activity (DLA) assay results indicated that K2 and K6 showed increased luciferase activity, with K6 showing significant fold changes (Fig. 3B).

To evaluate the change in KLF4 expression in vivo with the loss of $B m p 2$, we performed double immunofluorescence staining for KLF4 and SP7. As a result, a strong KLF4 signal was detected in the control bone trabeculae. Most of the KLF4-positive cells were SP7positive, indicating that KLF4 is specifically expressed in the $S p 7+$ lineage in bone (Fig. $3 \mathrm{C}$ ). In the BMP2-cKO mice, although the number of osteoblasts (marked by SP7) was reduced, KLF4 was barely detected in the mutant trabecular bone regions. These results suggest that Klf4 plays a positive role as a downstream TF of BMP2 during bone development. 
A

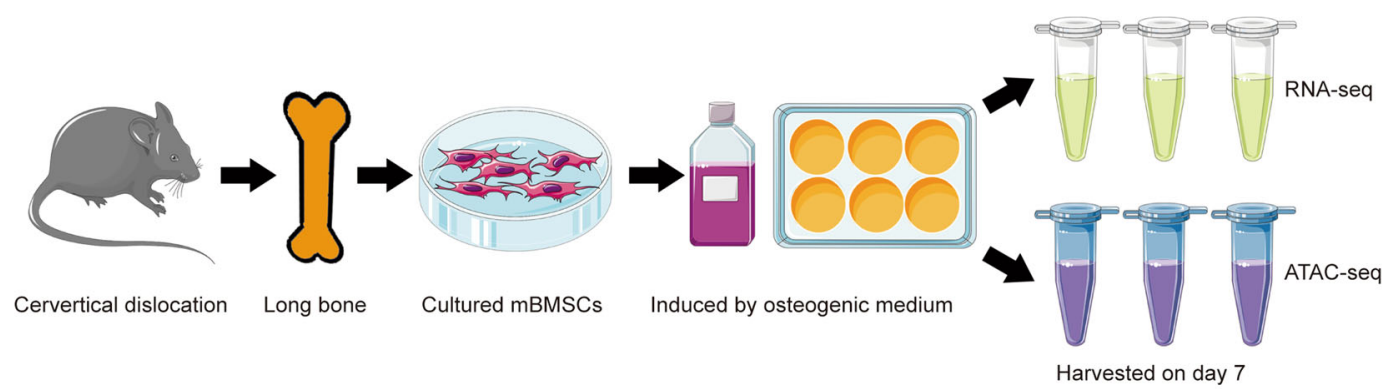

B

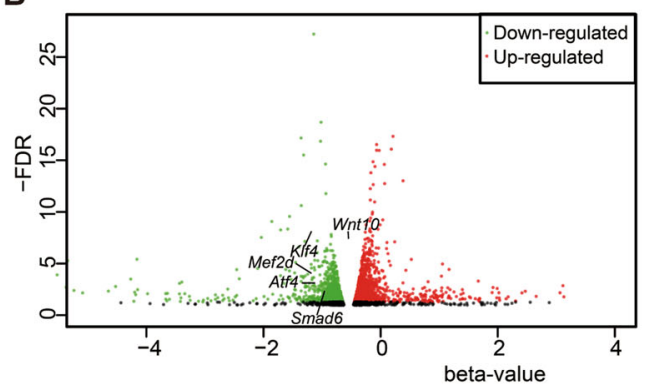

C

Selected Biological Process GO enrichment for down-regulated genes in BMP2-cKO mBMSCs

bone mineralization

osteoblast differentiation

cell cycle DNA replication -

DNA-dependent DNA replication

DNA replication

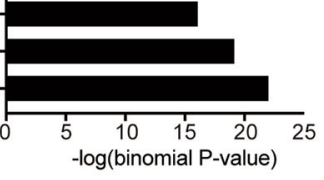

D

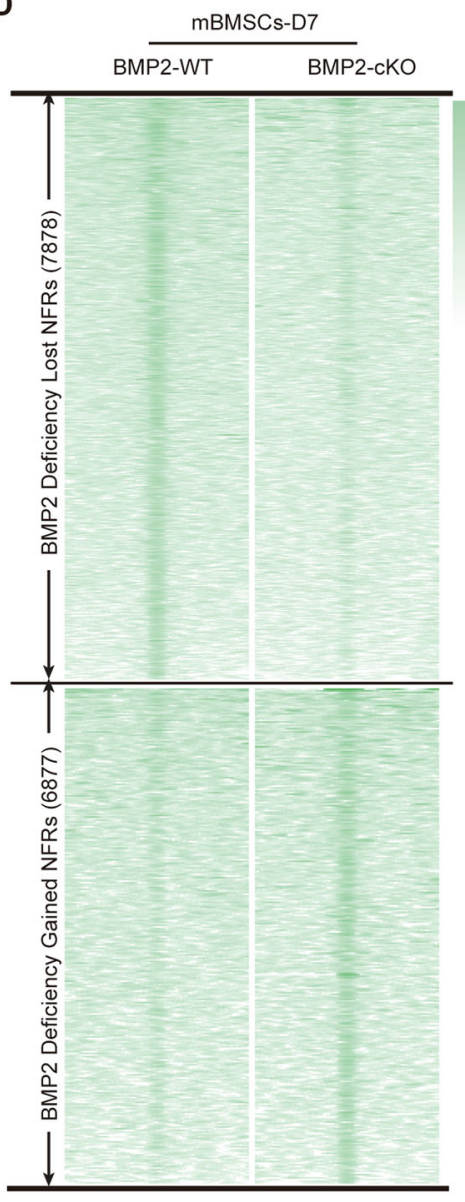

E

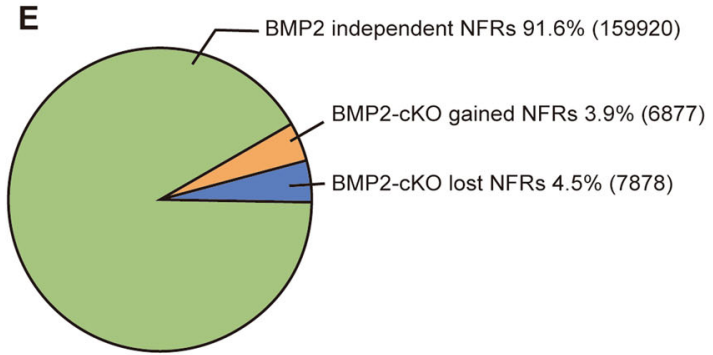

$\mathbf{F}$

Mouse phenotype enrichment for the nearby genes of Bmp2 deficiency lost NFRs

abnormal osteoclast cell number decreased osteoblast cell number abnormal tooth hard tissue morphology decreased bone strength abnormal trabecular bone thickness abnormal osteoblast cell number extramedullary hematopoiesis abnormal osteoblast physiology abnormal osteoblast morphology abnormal bone trabecula morphology abnormal bone volume

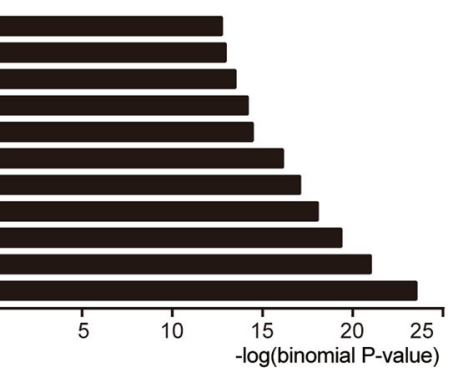

G

Motifs enriched in D7 BMP2-WT mBMSCs

\begin{tabular}{|c|c|c|c|}
\hline PWM & \%of targets & P-value & TF family \\
\hline IAATIACAGEC동 & $31.80 \%$ & $1 e-413$ & DLX \\
\hline 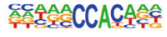 & $58.36 \%$ & $1 e-248$ & RUNX \\
\hline 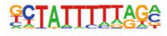 & $17.62 \%$ & $1 e-195$ & MADS \\
\hline 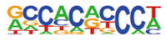 & $20.41 \%$ & $1 e-64$ & KLF \\
\hline ATGAGTCA & $13.36 \%$ & $1 e-44$ & bZIP \\
\hline
\end{tabular}

Fig. 2 (See legend on next page.) 
(see figure on previous page)

Fig. 2 Genomewide profile of BMP2-dependent genes and open chromatin landscape in osteoblast differentiation. A Cell sample preparation for RNA-seq and ATAC-seq. B Volcano plots of RNA-seq data, showing the genes up- or downregulated in BMP2-cKO osteogenic medium induced primarily-cultured mBMSCs. Green dots indicated the downregulated genes. Red dots indicated the upregulated genes. $n=3$. C GO enrichment of RNA-seq data for the downregulated genes in BMP2-CKO osteogenic medium induced primarily-cultured mBMSCs. D NFR summit-centred heat map of ATAC-seq signal in BMP2-WT and BMP2-cKO cells. $n=3$. E Pie chart showing the distribution of all 174,675 NFRs relative to gain and loss with deficiency of Bmp2. F Mouse phenotype enrichment assay for the nearby genes of Bmp2 deficiency lost NFRs revealed in ATACseq profiles. G Top five enriched motifs in deletion of Bmp2 lost NFRs. PWM position weighted matrix, TF transcription factors.
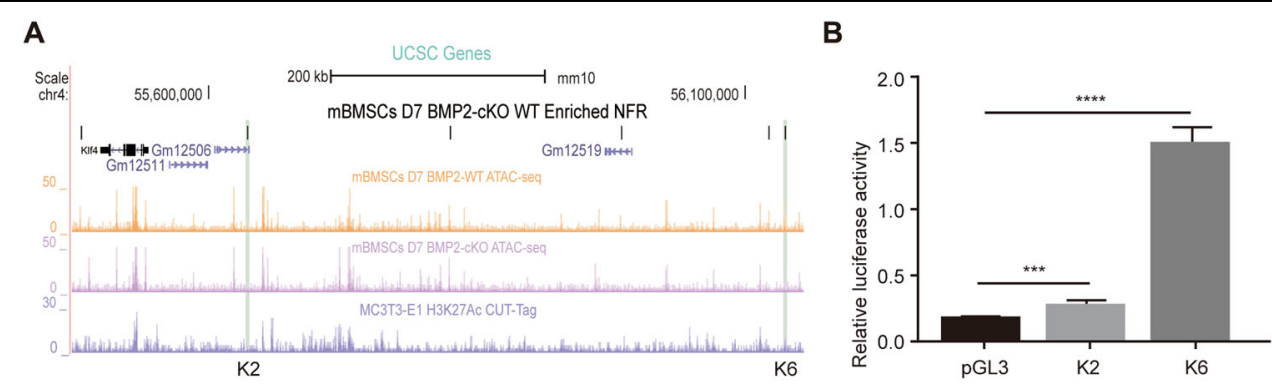

C

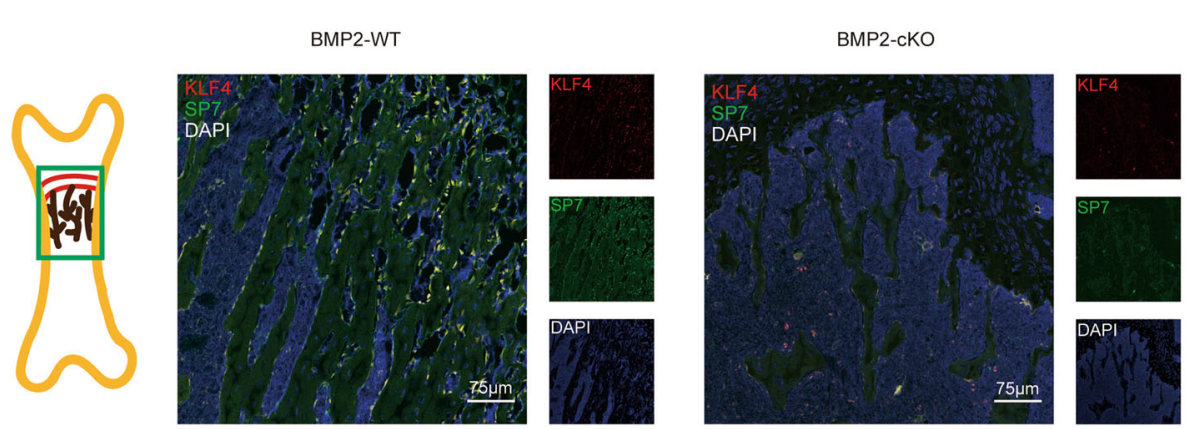

Fig. 3 Klf4 is a BMP2-dependent potential transcription factor governing osteoblast differentiation. A UCSC genome browser tracks showing representative replicate ATAC-seq and CUT\&Tag at the KIf4 locus. BMP2-dependent NFRs at Klf4 locus were named as K1 to K6. K2 and K6 are shaded in green. $\mathbf{B}$ Dual luciferase assay showing the relative enhancer activity of $\mathrm{K} 2$ and $\mathrm{K} 6$ in MC3T3-E1. $n=3,{ }^{* * *} P<0.001,{ }^{* * * *} P<0.0001$. C Double immunofluorescence staining of KLF4 and SP7 in 4-week-old Bmp2 ${ }^{\text {fx/fx }}$ (BMP2-WT) and Sp7-Cre; Bmp2 $2^{\text {fx/fx }}$ (BMP2-cKO) femurs. The image part was shown in the sketch on the left. $n=3$.

\section{Deletion of KIf4 in Sp7+ lineage induces decreased bone} mineralization and delayed endochondral ossification

To verify our hypothesis and elucidate the role of KLF4 in vivo, we deleted $K l f 4$ in the $S p 7+$ lineage and found that most of the homozygotes (Sp7-Cre; Klf4 $4^{\mathrm{fx} / \mathrm{fx}}$, KLF4-cKO) died immediately after birth. Skeletal staining at PN 0.5 confirmed decreased bone mineralization and delayed ossification in KLF4-cKO (Fig. 4A and S7B-E). The forelimbs (Fig. S7F) and hindlimbs (Fig. 4B) appeared normal in mineralization but were slightly shortened in KLF4-cKO.

Due to lethality of the homozygotes, we selected the control (Klf4 $4^{\mathrm{fx} / \mathrm{fx}}$, KLF4-WT) and heterozygotes (Sp7-Cre; $K l f 4^{\mathrm{fx} /+}$, KLF4-Het) for further study in subsequent experiments. At 8 weeks after birth, the KLF4-Het mice had smaller skeletons (Fig. 4C) and a decreased bone density (Fig. 4D-F) compared to the control littermates. H\&E staining confirmed that the KLF4-Het mice also showed a reduced trabecular bone number and a thinner cortical bone
(Fig. 4G). Notably, the KLF4-Het mice displayed abnormal incisors around 16 weeks after birth (Fig. S7G-J), which is similar to the craniofacial defects observed in the BMP2cKO mice. Overall, these results suggest that KLF4 plays a role in modulating skeletal development.

At 16 weeks before birth, the KLF4-Het mice exhibited histological phenotypes similar to BMP2-cKO mice (Fig. S8A, B). At 16 weeks, the trabecular bone number, cortical bone width, and growth plate length of KLF4-Het mice showed no obvious differences compared to the KLF4-WT mice (Fig. S8C). Safranin O staining revealed a shortened and disorganized hypertrophic zone in the KLF4-Het mice after two weeks (Fig. S8D).

Ki67 staining revealed that KLF4-Het mice showed reduced cell proliferation in the epiphyseal growth plate. The positive osteogenic-related markers (SP7 and OPN) domain indicated that the osteoblast number was reduced in KLF4-Het (Fig. 5A). These results demonstrate that the 


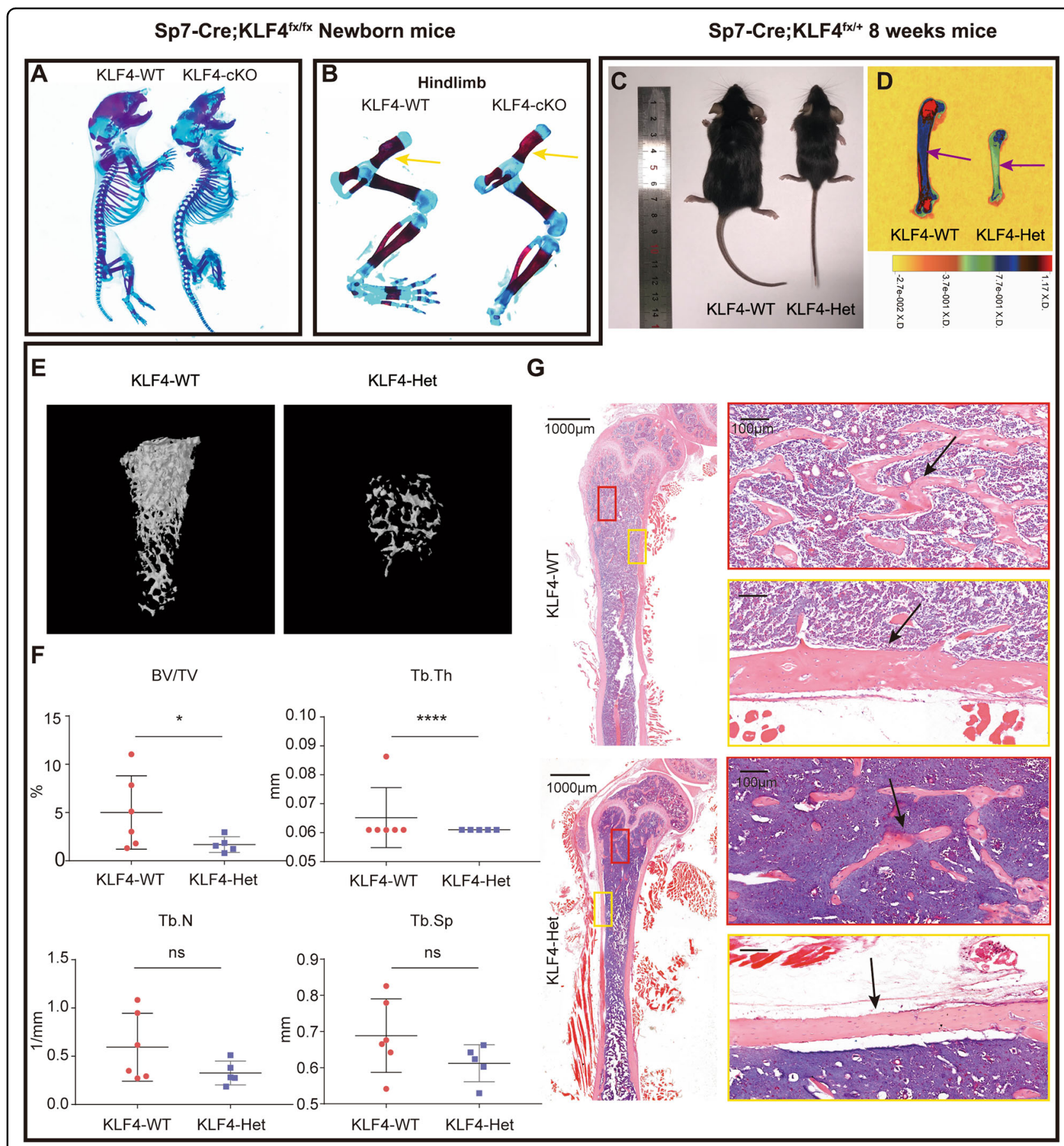

Fig. 4 SP7 + lineage KLF4-deficient mice suffered underdeveloped skeletons with decreased bone density. A Alizarin red/Alcian blue in newborn KIf4 ${ }^{\mathrm{fx} / \mathrm{fx}}$ (KLF4-WT) and Sp7-Cre; KIff ${ }^{\mathrm{fx} / \mathrm{fx}}$ (KLF4-CKO) mice. $n=3$. B Alizarin red/Alcian blue staining of hindlimbs from newborn KLF4-WT and KLF4-CKO mice. Yellow arrowheads indicated the hindlimbs of mutant mice were shorter but normal ossified. C Photographic analysis of 8-week-old KLF4-WT and Sp7-Cre; Klf4 ${ }^{\mathrm{f} /+}$ (KLF4-Het) mice. $n=6$. D Heat map of X-ray analysis of 8-week-old KLF4-WT and KLF4-Het mice. Purple arrowheads indicated decreased ossification in the KLF4-Het mouse femurs. $n=6$. E, F The micro-CT scans (E) and quantification (F) of 8-week-old KLF4-WT and KLF4-Het mouse femurs. Quantification data indicate bone volume/tissue volume (BV/TV), trabecular number (Tb.N), trabecular thickness (Tb.Th), and trabecular separation (Tb.Sp). $n=6$, ns = not statistically significant, ${ }^{*} P<0.05$, ${ }^{* * *} P<0.0001$. G H\&E staining of mouse femurs from 8-week-old KLF4WT and KLF4-Het mice. Black arrowheads indicated comparable trabecular bone number and cortical bone thickness. $n=3$. 
A
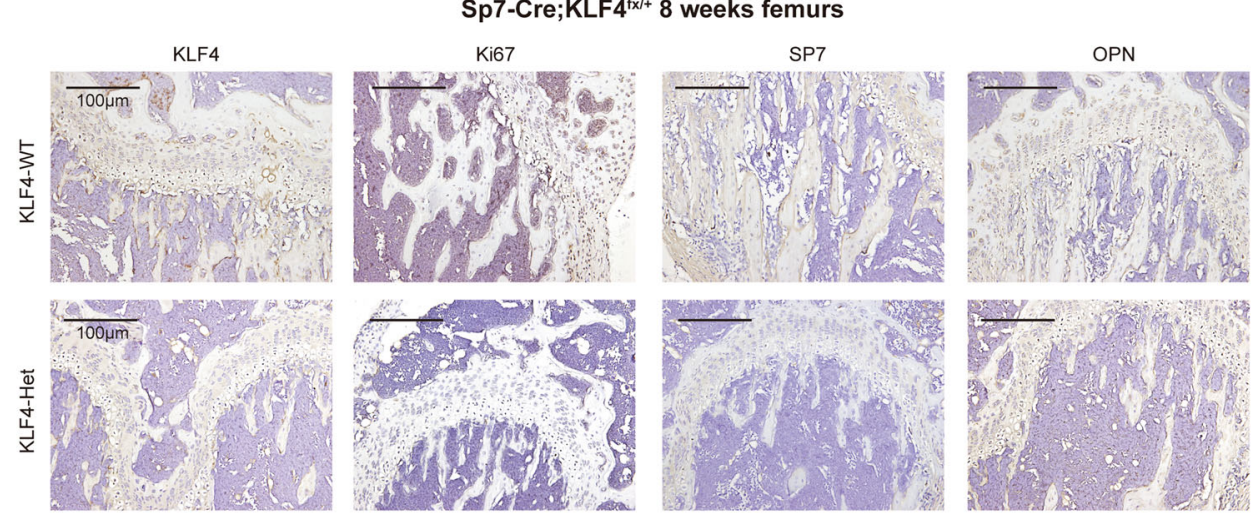

Sp7-Cre;KLF4 $4^{\mid \mathrm{x}+\mathbf{+}} 8$ weeks femurs

$\mathbf{B}$

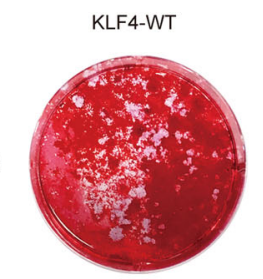

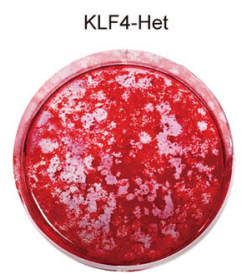
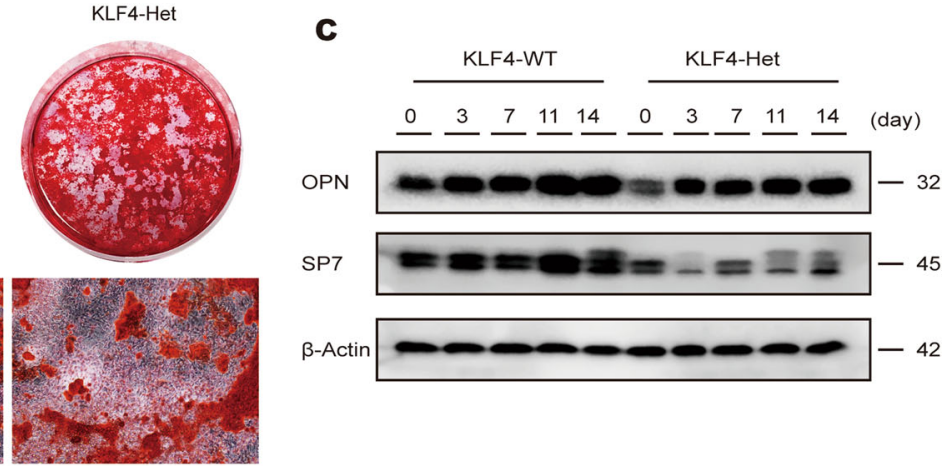

D

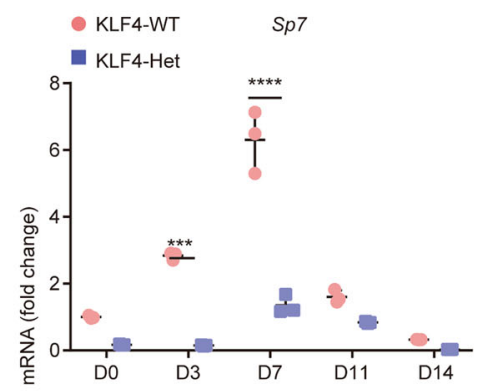

$\mathbf{E}$

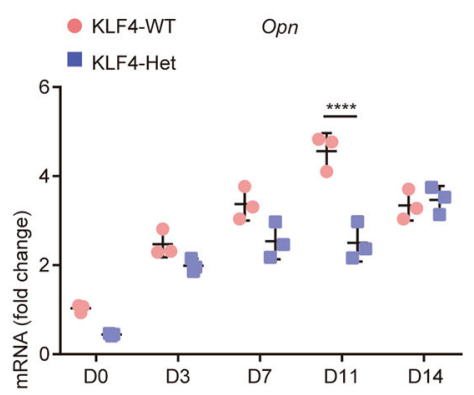

$\mathbf{F}$

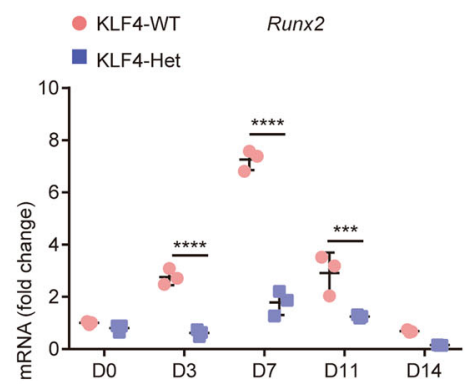

G

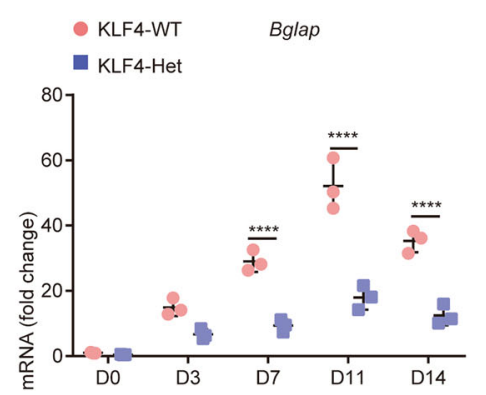

Fig. 5 Sp7-Cre; KIf4 $4^{\mathrm{f} /+}$ mice showed defective osteoblast formation in vivo and in vitro. A Immunohistochemistry (IHC) staining showed the expression of KLF4, Ki67, SP7, and OPN were decreased in 8-week-old Sp7-Cre; KIff ${ }^{f \times /+}$ (KLF4-Het) mouse femurs. $n=3$. B mBMSCs from KIff ${ }^{f \times / f x}$ (KLF4WT) and KLF4-Het mice were applied to Alizarin Red staining on day 14. $n=3$. C Western blot analysis of SP7 and OPN protein expression levels in mBMSCs. $\beta$-Actin was used as an internal control. $n=3$. D-G qRT-PCR analysis of Sp7 (D), Opn (E), Runx2 (F), and Bglap (G) mRNA expression levels in mBMSCs. Gapdh was used as an internal control. $n=3,{ }^{* * *} P<0.001,{ }^{* * * *} P<0.0001$. 
loss of Klf4 in the $S p 7+$ lineage impacts cellular proliferation and osteoblast differentiation in vivo. Taken together, these results indicate that KLF4 participates in osteoblast and chondrocyte maturation.

\section{Loss of Klf4 in Sp7+ cells affects osteoblast differentiation in vitro}

We then hypothesized that the deletion of Klf4 in the $S p 7+$ lineage also inhibited osteoblast differentiation in vitro. We harvested mBMSCs and induced with osteogenic medium. Klf4 expression in the KLF4-Het cells was confirmed (Fig. S9). During osteogenic induction, Klf4 remained at a low level in KLF4-Het, but increased markedly in KLF4-WT. During induction, the western blotting results indicated that the SP7 and OPN protein levels were downregulated in the KLF4-Het cells (Fig. 5C). qPCR analysis revealed that the mRNA levels of $S p 7, O p n$, Runx2, and Bglap remained at a low level in the KLF4-Het cells, in agreement with the protein results (Fig. 5D-G). Alizarin red staining showed that the amount of mineralized matrix was decreased in KLF4-Het (Fig. 5B). Hence, our results indicate that the deletion of $K l f 4$ in $S p 7+$ cells inhibits the osteoblast differentiation of mBMSCs in vitro.

\section{Correlating RNA-seq with ATAC-seq illustrates KIf4 downstream gene regulatory network}

Our KLF4-deficient transgenic mouse model supported the meaningful role of KLF4 in osteoblast differentiation. However, how KLF4 functions in osteoblast differentiation and whether it has cofactors to function as a transcriptional activator or repressor remains unknown. Using RNA-seq, we identified 1068 genes with a significant change $(P<0.01$ and $q<0.05)$ in their expression levels due to Klf4 deficiency. Among them, 440 genes were significantly downregulated by Klf4 knockdown $(\beta$-value $<0)$ and 628 genes were upregulated. The representative down- or upregulated genes were annotated in our RNA-seq results (Fig. 6A). Osteogenic marker genes, such as Alpl and Bglap, were found to be significantly decreased in the mutant group. Analysis of RNA-seq results revealed that the downregulated genes were significantly enriched for mineralization-related GO terms, such as ossification (GO: 0001503) and osteoblast differentiation (GO: 0001649) (Fig. 6B).

Next, we mapped the ATAC-seq fragments within the mouse genome. Discrepancies were observed in the density of the mapped reads (Fig. 6C). We found that KLF4 was required to maintain $\sim 8.8 \%$ (16607 NFRs) of the total NFRs in mBMSCs (Fig. 6D). On the other hand, most of Klf4 deficiency lost- and gained- NFRs were located at the distal regions (Fig. S10A, B). We analyzed the functional annotation of the nearby genes of Klf4 deficiency that lost NFRs and found that these NFRs were associated with metabolic-related biological processes
(Fig. S10C). The corresponding mouse phenotype mainly displayed skeletal anomalies and immune response, also observed in the mice affected by Klf4 (Fig. 6E). However, for Klf4 deficiency, the nearby genes were enriched, including those responsible for the epithelial-tomesenchymal transition and the negative regulation of the TGF- $\beta$ signaling pathway (Fig. S10D).

We also performed motif enrichment analysis on the 16607 KLF4-dependent NFRs, and discovered five highly enriched motifs. Surprisingly, except for the CEBPE motif family, the other top four motif families were the same as those observed in BMP2-dependent NFRs (Fig. 6F). But the 21,128 gained NFRs indicated discrepant enriched motifs compared with the lost NFRs (Fig. S10E). Using Cytoscape, we built a network connecting the major KLF4-WT enriched motifs with the best-matched candidate TFs. This network model suggests that most of the downstream TFs of KLF4 were enriched in RUNX and Smad4 motifs (Fig. S11). Furthermore, we globally compared BMP2-cKO lost NFRs and KLF4-Het lost NFRs and found that 4404 NFRs overlapped between the two groups (Fig. S12A). GO enrichment assay indicated that these overlapped NFRs were enriched in skeletal system development and osteoblast differentiation (Fig. S12B). Additionally, the top four enriched TF motifs were shared among the BMP2-cKO and KLF4-Het lost NFRs groups. A different motif, ZBTB, which was previously found to regulate lymphoid development and function ${ }^{37}$, was also enriched for their intersected NFRs (Fig. S12C). These results indicated that KLF4 and BMP2 regulate a large set of similar NFRs through a same set of downstream TFs.

To elucidate the epistasis between BMP2 and KLF4 during osteoblast differentiation, we added BMP2 in KLF4-Het mBMSCs to determine whether BMP2 could rescue Klf4 expression in vitro. As a result, KLF4, SP7, and OPN were found to be upregulated in KLF4-Het cells treated with BMP2 for 7 days, and KLF4 and SP7 were comparable to those in KLF4-WT (Fig. 8A). Alizarin red staining showed increased calcified matrix formation after 7 days of stimulation in KLF4-Het group with addition of BMP2 (Fig. 8B). From these results, we concluded that haploinsufficiency of KLF4 could be partially rescued by BMP2 in vitro.

The results of our integrated methods show that KLF4 regulates osteoblast differentiation with similar enriched motif as BMP2, further targeting downstream osteoblastrelated genes. KLF4 was identified as a downstream member of BMP signaling.

\section{RUNX2 as a cofactor with KLF4 synchronously governs osteoblast differentiation}

Based on motif enrichment analysis, the Klf4 deficiency lost NFR-enriched motifs was critical for osteoblast differentiation. Next, we investigated whether these 
A

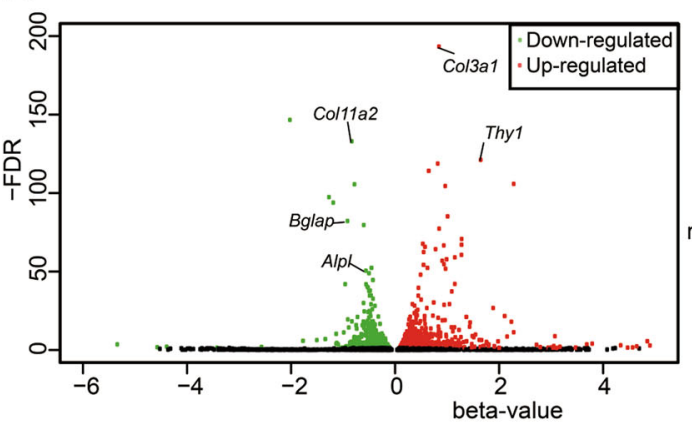

C

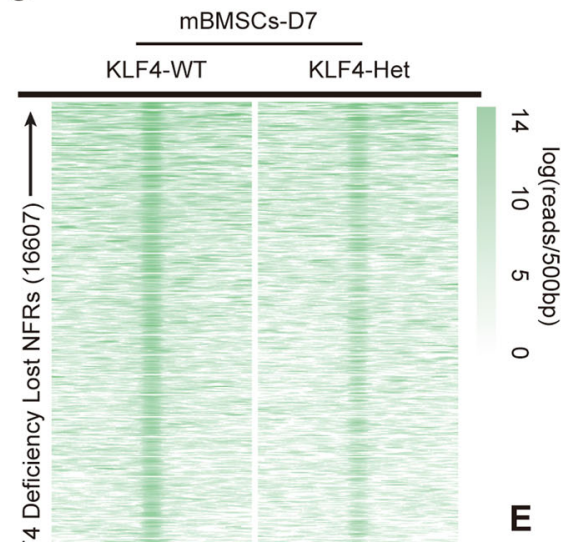

B

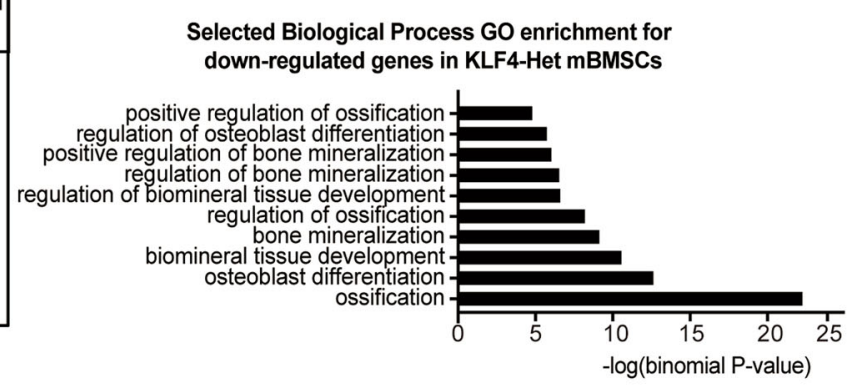

D

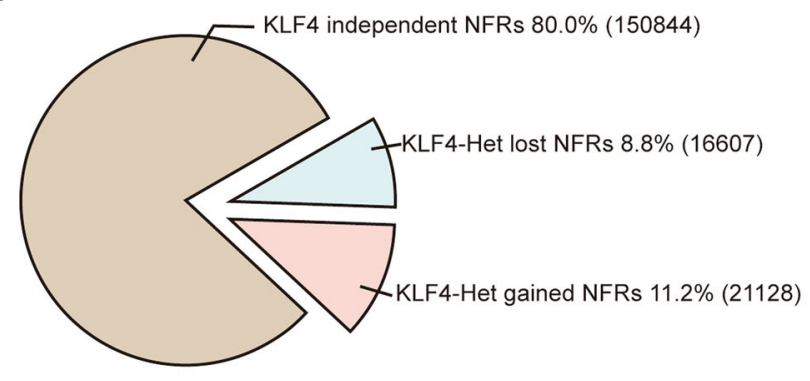

E Mouse phenotype enrichment for the nearby genes of KLF4 deficiency lost NFRs

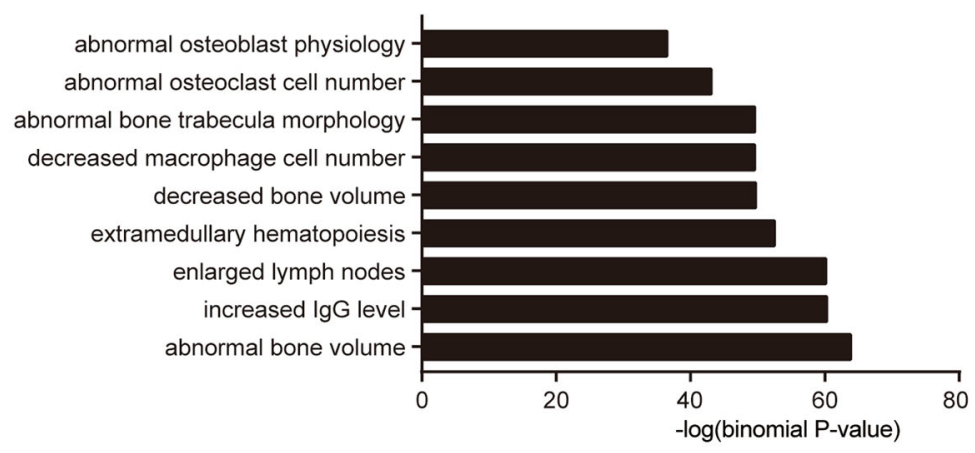

$\mathbf{F}$

Motifs enriched in D7 KLF4-WT mBMSCs

\begin{tabular}{|c|c|c|c|}
\hline PWM & \%of targets & P-value & TF family \\
\hline IAATTACAGGCST & $19.33 \%$ & $1 \mathrm{e}-765$ & DLX \\
\hline 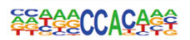 & $44.71 \%$ & $1 e-673$ & RUNX \\
\hline G्र्रTAIIIIAGA & $16.29 \%$ & $1 e-297$ & MADS \\
\hline 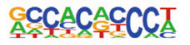 & $21.96 \%$ & $1 e-169$ & KLF \\
\hline ATTCCEAAAC & $14.69 \%$ & $1 \mathrm{e}-112$ & CEBPE \\
\hline
\end{tabular}

Fig. 6 Genomewide profile of KLF4-dependent genes and open chromatin landscape in osteoblast differentiation. A Volcano plots of RNAseq data, showing the genes up- or downregulated in KLF4-Het osteogenic medium induced primarily-cultured mBMSCs. Green dots indicated the downregulated genes. Red dots indicated the upregulated genes. $n=3$. B GO enrichment of RNA-seq data for the downregulated genes in KLF4-Het osteogenic medium induced primarily-cultured mBMSCs revealed in RNA-seq profiles. C NFR summit-centred heat map of ATAC-seq signals in KLF4WT and KLF4-Het cells. $n=3$. D Pie chart showing the distribution of all 188,579 NFRs relative to gain and loss with deficiency of KLF4. E Mouse phenotype enrichment assay for the nearby genes of KIf4 deficiency lost NFRs revealed in ATAC-seq profiles. $\mathbf{F}$ Top five enriched motifs in deletion of KIf4 lost NFRs. PWM position weighted matrix, TF transcription factors. 
members could regulate different or the same subset of osteogenesis genes dependent on KLF4. Hence, we examined the enrichment of the genes near the NFRs occupied with KLF motif only or a combination of KLF and other motifs. When only the KLF motif was occupied, biological processes were not associated with skeletal system development (Fig. 7A). However, when KLF was "cobound" with RUNX, DLX, MADS, or CEBPE motifs, most of the nearby genes of the related NFRs manifested significant enrichment in skeletal system development (Figs. 7B and S13A-C). These results suggest that Klf4 itself does not directly regulate skeletal development and requires other osteogenesis-related transcription factors as cofactors to drive skeletal development.

Because the RUNX motifs accounted for $44.17 \%$ of the motifs directly affected by KLF4 in mBMSCs, and previous studies have found that KLF4 is physically associated with RUNX2 $2^{38,39}$, we evaluated their physical binding results (Fig. S13D). Therefore, we focused on the NFRs with both KLF- and RUNX-motif-relevant genes. Considering the KLF4-deficient RNA-seq results, Alpl and Bglap were selected as downstream targets. And we chose two emblematic NFRs from the two loci separately to verify their transcription activity (Fig. 7C, F). For the Alpl locus, both representative NFRs were found on the Alpl gene sequence A6 (mm10: chr4:137748653-137749194) and A9 (mm10: chr4:137768825-137769369). The DLA assay results indicated that A6 and A9 exhibited increased luciferase activity with no obvious fold changes (Fig. 7D). Double immunofluorescence staining showed a strong ALPL and SP7 signal in the KLF4-WT bone trabeculae, but a weak ALPL and SP7 signal in the KLF4-Het group (Fig. 7E). For the Bglap locus, we chose B1 (mm10: chr3: 88372858-88373399) and B2 (mm10: chr3:8837857788379119). We found that both B1 and B2 exhibited increased luciferase activity, and B1 exhibited at least a 10 -fold change. We evaluated B1 as a functional enhancer in the presence of KLF motifs and found that the deletion of KLF motifs in B1 reduced enhancer activity (Fig. 7G). Collectively, these results indicate that KLF motifs are essential for Bglap enhancer activity. Double immunofluorescence staining showed a strong BGLAP signal in the KLF4-WT bone trabeculae, and the BGLAP-positive region was larger than SP7positive. However, both the BGLAP- and SP7-positive regions were reduced in KLF4-Het bone (Fig. 7H). Taken together, our results suggested that KLF4 could "cobond" with Runx2 to further regulate downstream genes, including Alpl and Bglap.

\section{Discussion}

To illustrate the BMP2-dependent gene regulatory network in osteoblast differentiation, we selectively ablated $B m p 2$ in $S p 7+$ lineage in vivo and then analyzed its function in vitro. We found $B m p 2$ in $S p 7+$ cells was dispensable for embryonic skeletal development but essential for postnatal endochondral ossification. Our findings are consistent with those of previous stu$\operatorname{dies}^{12,40,41}$. Due to the inhibition of postnatal bone development via changes to osteoblastogenesis, we analyzed the critical downstream TFs and focused on the function of Klf4 in osteoblast differentiation, and our findings highlighted Klf4 as a novel transcription factor in bone development.

KLF4 is known for its role in the maintenance of stemness in pluripotent stem cells ${ }^{42}$. KLF4 also functions in various cell differentiation processes, such as epithelial differentiation ${ }^{43}$ and adipogenesis ${ }^{44}$. Interestingly, our group has spent many years studying the role of KLF4 in odontoblast differentiation. Recently, we deleted Klf4 in odontoblast cells with Wnt1-Cre and found that Klf4 deficiency affected dentin deposition and formation ${ }^{45}$. In the present study, we specifically deleted Klf4 in the $S p 7+$ lineage to study the in vivo role of KLF4. Unexpectedly, the deletion of Klf4 in the Sp7+ lineage resulted in postnatal death. As such, we selected heterozygotes as our main targets. KLF4-Het mice exhibited osteogenesis defects analogous to those observed in BMP2-cKO mice, indicating that KLF4 are necessary in osteoblasts. Our RNA-seq and ATAC-seq results also elucidated the functions of KLF4 in bone formation. We reported KLF4 itself was not sufficient to prime osteoblast differentiation, but rather acted with RUNX2 as a cofactor to activate potential enhancers and regulate downstream genes, to determine osteoblast differentiation and osteogenesis. Our results, therefore, markedly supported the multidisciplinary perspective of BMP signaling and established unique insights into the role of KLF4 during bone development.

The current study presents some controversial results compared to similar previous studies. A group culturing mBMSCs from BMP2-WT and BMP2-cKO mice in osteogenic medium suggested that $B m p 2$ is dispensable for osteoblast differentiation in the $S p 7+$ lineage ${ }^{19}$. However, our results indicate opposite conclusion. This distinction may be due to differences in the composition of the osteogenic medium. In the present study, $10 \mathrm{nmol} /$ $\mathrm{L}$ of dexamethasone (Dex) was added to the osteogenic medium, while other studies did not. Dexamethasone promoted the expression of osteogenic markers, such as RUNX2, SP7, and OPN in vitro ${ }^{46-49}$. The deletion of Bmp2 in the $S p 7+$ lineage may block the activation of Dex-induced osteo-related signaling pathways. In addition, using Col1 $\alpha 1$-Cre; $B m p 2^{2^{\mathrm{fx} / \mathrm{fx}}}$ mice, other researchers demonstrated that the $B m p 2$ in osteoblasts is required for terminal differentiation ${ }^{50}$. It is worth noting that Klf4 is a controversial transcription factor. A previous study reported that KLF4 is a negative regulator of osteoblast 


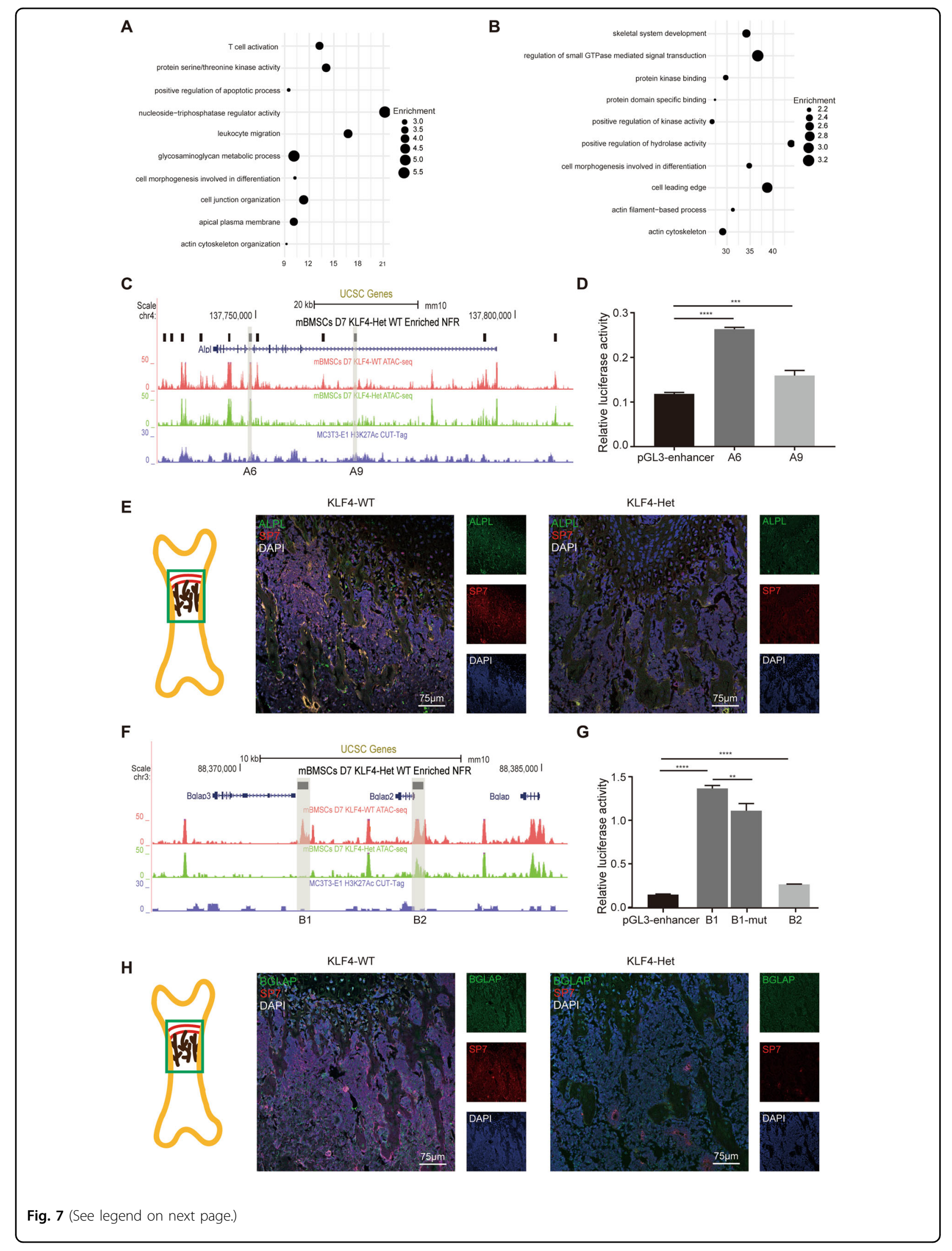


(see figure on previous page)

Fig. 7 RUNX2 as a cofactor with KLF4 governing osteoblast differentiation. A Dot plot showing the GO enrichment assay for the nearby genes of only KLF motif occupied in KLF4 deficiency lost NFRs. B Dot plot showing the GO enrichment assay for the nearby genes of KLF occupied with RUNX motif in Klf4 deficiency lost NFRs. C UCSC genome browser tracks showing representative replicate ATAC-seg and CUT\&Tag at the Alp/ locus. KLF4-dependent NFRs at Alpl locus were named as A1 to A11. A6 and A9 are shaded in gray. D Dual luciferase assay showing the relative enhancer activity of A6 and A9 in MC3T3-E1. $n=3,{ }^{* * *} P<0.001$, ${ }^{* * *} P<0.0001$. E Double immunofluorescence staining of ALPL and SP7 in 4-week-old KIf4 ${ }^{\mathrm{fx} / \mathrm{fx}}$ (KLF4-WT) and Sp7-Cre; KIf4 ${ }^{\mathrm{f} /+}$ (KLF4-Het) femurs. The image part was shown in the sketch on the left. $\mathrm{n}=3$. F UCSC genome browser tracks showing representative replicate ATAC-seq and CUT\&Tag at the Bglap locus. KLF4-dependent NFRs at Bglap locus were named as B1 to B2. B1 and B2 are shaded in gray. G Dual luciferase assay showing the relative enhancer activity of $B 1, B 2$ and $B 1$ without KLF motif in $M C 3 T 3-E 1 . n=3$, **P $<0.01$, ${ }^{* * * * P}<0.0001$. H Double immunofluorescence staining of BGLAP and SP7 in 4-week-old KLF4-WT and KLF4-Het femurs. The image part was shown in the sketch on the left. $n=3$.

differentiation in vitro and in vivo ${ }^{39}$, using Col1 $\alpha$-Cre; $K l f 4^{\mathrm{fx} / \mathrm{fx}}$ mice. Using different Cre mouse lines, we obtained different conclusions about KLF4. Col1 $\alpha 1$-Cre mainly targets mature osteoblasts, while Sp7-Cre mainly targets immature osteoblasts ${ }^{51}$. These results suggest that, during embryogenesis and postnatal bone development, KLF4 plays temporal- and spatial-specific roles in the process of bone development. However, we were unable to elucidate the precise function of KLF4 during the whole cell differentiation process. Based on varied conditions in vivo, it may activate tissue-specific enhancers and perform a spatial-temporal function.

Members of the BMP superfamily affect almost all aspects of bone, cartilage, and joint biology. Defective BMP superfamily molecules are the underlying cause of human skeletal pathologies. Thus, modulating BMP superfamily signaling is a potential method for inducing stem cells for tissue repair. KLF4-Het mice exhibited the consistency of the long bone phenotype like BMP2-cKO mice, but chondrocyte defects were milder. In addition, KLF4-cKO mice exhibited more significant cranial dysplasia. Although our results showed that Klf4 was controlled by BMP signaling, other key signaling pathways must participate to direct the regulation of $K l f 4$ in skeletal development. Further study is necessary to determine the branched regulatory network in the process of endochondral ossification and intramembranous ossification, which may provide new therapeutic approaches for skeletal system disease.

To summarize, we propose Klf4 as a BMP2-dependent novel transcription factor in osteoblast differentiation (Fig. 8C). When the extracellular signal molecule BMP2 binds to the transmembrane type I and type II receptors, the BMP signaling pathway is activated. BMP signaling synergizes osteogenic transcription factors, such as Runx2 and Klf4. KLF4 itself was not able to prime osteoblast differentiation but could "cobound" with osteogenesisenriched TFs (RUNX, DLX, MADS, and CEBPE) to regulate osteoblast differentiation. Among them, RUNX2, a coactivator with KLF4, regulates the expression of Alpl and Bglap. Collectively, our findings indicate the existence of a genome-wide BMP2-dependent gene regulatory network and provide novel insights into the role of KLF4 in osteoblast differentiation.

\section{Materials and methods}

\section{Transgenic mice generation and maintenance}

All mouse-related experimental protocols were approved by the Institutional Animal Care and Use Committees at the School and Hospital of Stomatology of Wuhan University (protocol no. S07917110B). B6;129S4$\mathrm{Bmp} 2^{\mathrm{tm} 1 \mathrm{Jm}} / \mathrm{J}$ (hereafter $\mathrm{Bmp} 2^{\mathrm{fx} / \mathrm{fx}}$ ) mice ${ }^{23,52}$ and B6.Cg$\mathrm{Tg}(\mathrm{Sp} 7$-tTA,tetO-EGFP/cre)1Amc/J (hereafter Sp7-Cre) mice $^{18}$ were purchased from Jackson Laboratory, B6.129S6-Klf $4^{\mathrm{tm} 1 \mathrm{Khk}} / \mathrm{Mmmh}\left(K l f 4^{\mathrm{fx} /+}\right)$ mice $^{53}$ were purchased from the Mutant Mouse Regional Resource Centers (MMRRC). To rule out potential defects in Sp7-Cre mice as previously reported ${ }^{54}$, we also examined every Sp7-Cre littermate. All mice were maintained with good ventilation and kept on a standard diet and a 12-h day/ night rhythm.

\section{Immunohistochemistry}

The $6-\mu \mathrm{m}$ paraffin sections were deparaffinized, rehydrated, and antigen-retrieved with gastric enzyme. The sections were incubated with anti-BMP2 (ab6285; Abcam, Cambridge, MA, USA), anti-SP7 (sc-393325; Santa Cruz Biotechnology, Santa Cruz, CA, USA), anti-OPN (0806-6; HuaAn Biotech, Hangzhou, Zhejiang, China), anti-KLF4 (11880-1-AP; Proteintech, Wuhan, Hubei, China), and anti-Ki67 (ET1609-34; HuaAn Biotech) overnight at $4{ }^{\circ} \mathrm{C}$. After incubation with horseradish peroxidase-conjugated secondary antibody, the sections were visualized using a diaminobenzidine substrate kit (DAKO, Carpinteria, CA, USA). The immunostained sections were counterstained with hematoxylin.

\section{Double immunofluorescence staining}

The 6- $\mu \mathrm{m}$ paraffin sections were deparaffinized, rehydrated, antigen-retrieved, blocked, and then incubated with primary antibodies overnight at $4{ }^{\circ} \mathrm{C}$. The antibodies included anti-KLF4, anti-SP7, anti-BGLAP (ab93876; Abcam), and anti-ALPL (AF2910; R\&D Systems, Minneapolis, MN, USA). The sections were then 


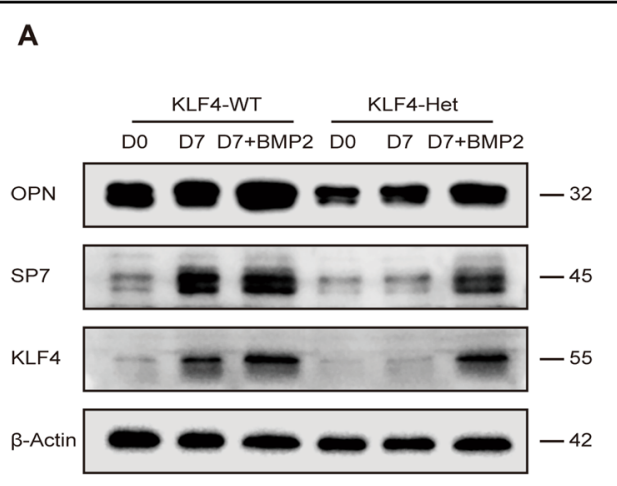

B
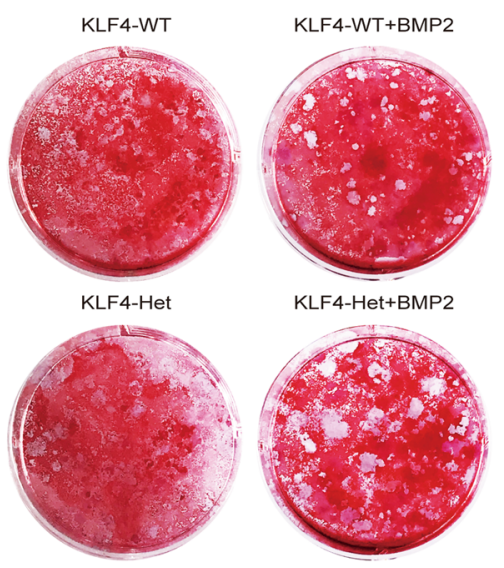

C

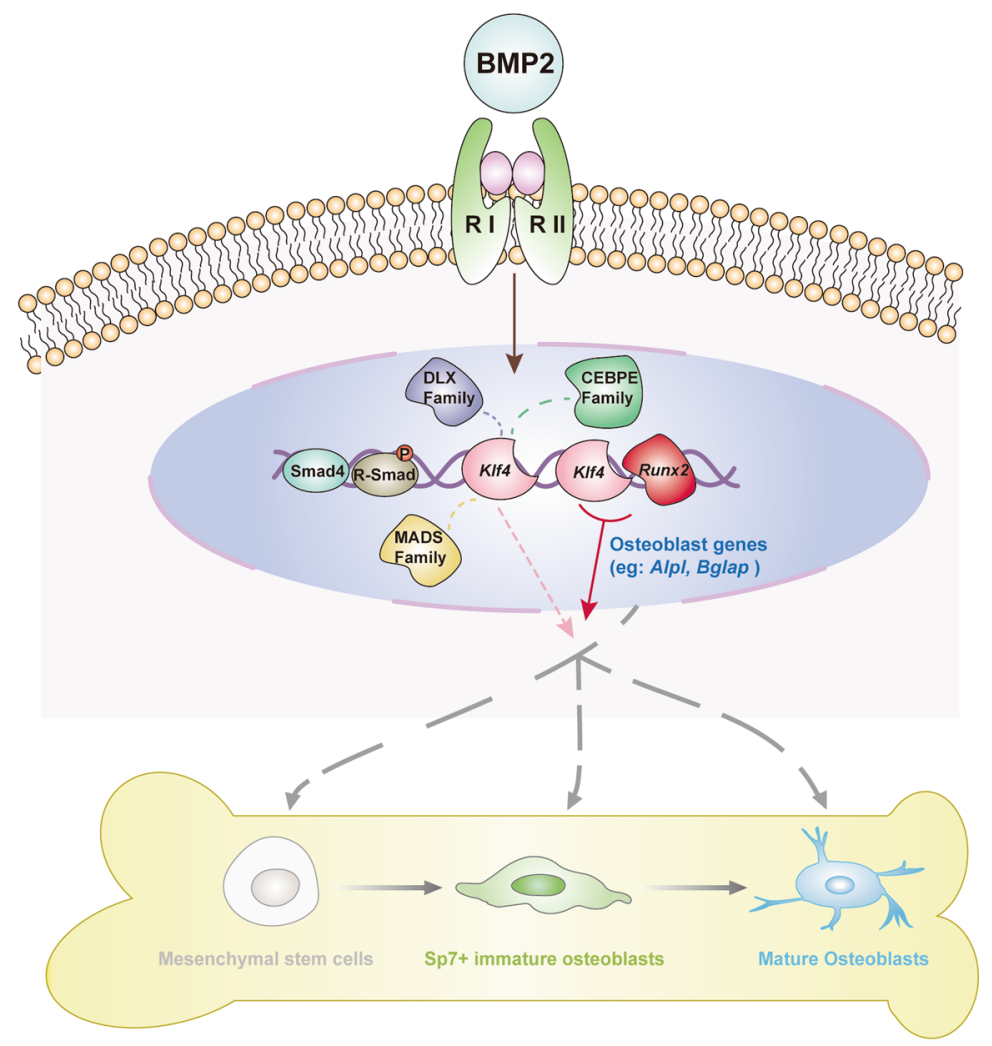

Fig. 8 Klf4 is a BMP2-dependent transcription factor governing osteoblast differentiation. A Western blot analysis of KLF4, SP7, and OPN protein expression levels in osteogenic medium induced primarily-cultured mBMSCs with or without BMP2 treatment. $\beta$-Actin was used as an internal control. $n=3$. B BMSCs from KIf4 ${ }^{\mathrm{f} / \mathrm{fx}}$ (KLF4-WT) and Sp7-Cre; KIff4 ${ }^{\mathrm{f} / \mathrm{f}}$ (KLF4-Het) mice with or without BMP2 treatment were applied to Alizarin Red staining on day 7. $n=3$. C During the osteoblast differentiation, BMP superfamily signal molecules act locally on target cells in which targeted transcription factors switch is turned on. KIf4 as a downstream transcription factor involved during mesenchymal stem cells to mature osteoblasts differentiation. KIf4 itself could not prime whole differentiation process, but it could interact with coactivators such as RUNX2 (or DLX, MADS, CEBPE family TFs) to initiate the expression of osteogenic marker genes (eg: Alpl and Bglap) further governing osteogenesis and osteoblast maturation.

incubated with Alexa Fluor 488- or 594-conjugated secondary antibodies (Jackson Immuno Research, West Grove, PA, USA). Finally, the stained sections were mounted with DAPI.

\section{Quantitative real-time PCR assay}

Total RNA from cultured cells was isolated using HP Total RNA kit (Omega Bio-Tech, Norcross, Georgia, USA). Reverse transcription was performed using 
RevertAid RT Reverse Transcription Kits (Thermo Fisher Scientific, Waltham, MA, USA). Quantitative real-time PCR (qRT-PCR) was performed using the CFX96 Touch Real-Time PCR Detection PCR System (BIO-RAD, Berkeley, CA, USA) with SYBR Premix Ex Taq (Takara, Kusatsu, Gunma, Japan).

\section{Western blot analysis}

Cells were lysed in a buffer containing protease inhibitors. Total proteins were harvested and equal amounts of protein were resolved by $12 \%$ SDS/PAGE and then transferred onto a PVDF membrane. The membranes were incubated with the primary antibody for SP7, OPN, KLF4 (ab106629; Abcam), or $\beta$-Actin (PM053; MBL, Nagoya, Aichi-ken, Japan) at $4^{\circ} \mathrm{C}$ overnight. After incubation with horseradish peroxidase-labeled IgG. ECL solution (Pierce Biotechnology, Rockford, IL, USA) was used for signal visualization.

\section{RNA-seq library generation}

Cultured mBMSCs were induced with osteogenic medium for 7 days, and $3 \mu \mathrm{g}$ of total RNA was isolated using an RNeasy mini kit (Qiagen, Valencia, CA, USA). Genomic DNA was digested using Turbo-DNaseI using DNasel (Promega, San Luis Obispo, CA, USA). The library quality was assessed using a DNA 1000 kit (5067-1504; Agilent) on a Bioanalyzer 2100 (Agilent, Santa Clara, CA, USA). NEBNext Ultra RNA Library Prep Kit (New England Biolabs, Ipswich, MA, USA) was used to generate and index RNA-seq libraries. 150-bp-pairedend sequencing was performed using a Hiseq $X$ Ten sequencer (Illumina, San Diego, CA, USA, provided by Annoroad Genomics Company (China)). Then, sequencing reads were pseudo-aligned to the $\mathrm{mm} 10$ mouse genome.

\section{ATAC-Seq library preparation}

Library preparation was performed using our previously reported protocol ${ }^{45}$. Briefly, mBMSCs were cultured in osteogenic medium for 7 days, and $\sim 50,000$ cells were used for the library. Cells were washed in cold PBS, resuspended in cold lysis buffer $(10 \mathrm{mM}$ Tris- $\mathrm{HCl}$ at $\mathrm{pH}$ 7.4, $10 \mathrm{mM} \mathrm{NaCl}, 3 \mathrm{mM} \mathrm{MgCl} 2,0.1 \% \mathrm{NP}-40$ ). The tagmentation reaction was performed using $\mathrm{Tn} 5$ transposase (TD501; Vazyme, Nanjing, Jiangsu, China). The DNA library was eluted using a purification kit (Mini Elute kit; Qiagen) to obtain purified DNA, which was amplified and barcoded with the NEBNext High-Fidelity $2 \times$ PCR Master Mix (New England Biolabs). DNA fragments were purified using AMPure beads (Beckman Coulter, Brea, CA, USA), and the library quality was analyzed using BioAnalyzer 2100. Sequencing was performed using a HiSeq X Ten sequencer (Illumina, provided by Annoroad Genomics Company (China)).

\section{Plasmid constructs and dual luciferase report assay}

All candidate enhancer elements described were cloned using mouse genomic DNA. Wild-type or mutant DNA fragments were cloned into the pGL3-basic plasmids and Sangon sequencing was performed to validate the sequence. MC3T3-E1 cells were seeded in 24-well plates and transfected with $1 \mu \mathrm{g}$ of reporter luciferase vector and $200 \mathrm{ng}$ of pRL-TK using Lipofectamine 2000 reagent (Invitrogen). After $48 \mathrm{~h}$ of transfection, the cells were harvested and the luciferase activity was measured using the Dual-Luciferase Reporter Assay System (Promega), according to the manufacturer's instructions. GloMax 20/20 Luminometer (Promega) was used to evaluate the ratio between firefly and Renilla activity.

\section{Sample processing}

Each experiment was performed at least three independent replicates. All data represented biological replicates. Sample size of animals was at least three based on preliminary tests. Wild-type samples were collected randomly and conditional knockout samples were collected based on obvious phenotype deficiency. No samples were excluded from the analyzation. Data was analyzed blind.

\section{Statistical analysis}

Except for the high-throughput sequencing results, data are presented as the mean \pm standard deviation (SD; $n>$ 3). One-way analysis of variance (ANOVA) was used to compare multiple group comparisons, and Student's t test was applied to compare two groups. $P<0.05$ was considered statistically significant.

\section{Acknowledgements}

We thank the Supercomputing Center of Wuhan University for providing numerical calculations in this paper.

\section{Funding}

This work was supported by grants from National Natural Science Foundation of China (No. 81420108011, 81771066, and 8207110) to Z. Chen and grants from the Young Elite Scientist Sponsorship Program by CAST (No. 2017QNRC001) and from the National Natural Science Foundation of China (No. 81771057 and 82071077 ) to H. Liu.

\section{Author details \\ ${ }^{1}$ State Key Laboratory Breeding Base of Basic Science of Stomatology (Hubei- MOST) and Key Laboratory for Oral Biomedicine of Ministry of Education (KLOBM), School and Hospital of Stomatology, Wuhan University, Wuhan, China. ${ }^{2}$ Department of Developmental Dentistry, University of Texas Health Science Center, San Antonio, TX, USA. ${ }^{3}$ Department of Periodontology, School and Hospital of Stomatology, Wuhan University, Wuhan, China}

\section{Author contributions}

S.Y.: transgenic mice breeding, experimental performing, data analysis and interpretation, and manuscript drafting. J.G.: transgenic mice breeding and data analysis. Z.S., C.L., H.T., and Q.Z.: data acquisition and interpretation. Y.C. and H.Z.: experimental performing and data analysis. Y.L.: manuscript revision. S.C.: study conception and data interpretation. H.L. and Z.C.: study conception and design, data interpretation, and manuscript revision. All authors read and approved the final paper. 


\section{Data availability}

All sequencing profiles, including RNA-seq and ATAC-seq, were uploaded to the China National Center for Bioinformation (CNCB). The datasets are available in the GSA (accession NO. CRA003341).

\section{Conflict of interest}

The authors declare no competing interests.

\section{Ethics statement}

All animal experiments were approved by the Animal Ethics Committee of School of Stomatology, Wuhan University (protocol no. S07917110B).

\section{Publisher's note}

Springer Nature remains neutral with regard to jurisdictional claims in published maps and institutional affiliations.

Supplementary information The online version contains supplementary material available at https://doi.org/10.1038/s41419-021-03480-7.

\section{Received: 16 November 2020 Revised: 21 January 2021 Accepted: 22} January 2021

Published online: 19 February 2021

\section{References}

1. Hall, B. K. \& Miyake, T. The membranous skeleton: the role of cell condensations in vertebrate skeletogenesis. Anat. Embryol. 186, 107-124 (1992).

2. Reddi, A. H. Cell biology and biochemistry of endochondral bone development. Collagen Relat. Res. 1, 209-226 (1981).

3. Olsen, B. R., Reginato, A. M. \& Wang, W. Bone development. Annu. Rev. Cell Dev. Biol. 16, 191-220 (2000).

4. Nakashima, K. \& de Crombrugghe, B. Transcriptional mechanisms in osteoblast differentiation and bone formation. Trends Genet. 19, 458-466 (2003).

5. Zabidi, M. A. \& Stark, A. Regulatory enhancer-core-promoter communication via transcription factors and cofactors. Trends Genet. 32, 801-814 (2016).

6. Bemer, M., van Dijk, A. D. J., Immink, R. G. H. \& Angenent, G. C. Cross-family transcription factor interactions: an additional layer of gene regulation. Trends Plant Sci. 22, 66-80 (2017)

7. Brahma S., Henikoff S. Epigenome regulation by dynamic nucleosome unwrapping. Trends Biochem. Sci. 45, 13-26 (2019).

8. Hogan, B. L. Bone morphogenetic proteins: multifunctional regulators of vertebrate development. Genes Dev. 10, 1580-1594 (1996).

9. Sanchez-Duffhues, G., Hiepen, C., Knaus, P. \& Ten Dijke, P. Bone morphogenetic protein signaling in bone homeostasis. Bone 80, 43-59 (2015).

10. Salazar, V. S., Gamer, L. W. \& Rosen, V. BMP signalling in skeletal development, disease and repair. Nat. Rev. Endocrinol. 12, 203-221 (2016).

11. Reddi, A. H. Bone morphogenetic proteins: an unconventional approach to isolation of first mammalian morphogens. Cytokine Growth Factor Rev. 8, 11-20 (1997)

12. Bandyopadhyay, A. et al. Genetic analysis of the roles of BMP2, BMP4, and BMP7 in limb patterning and skeletogenesis. PLoS Genet. 2, e216 (2006).

13. Wu, M., Chen, G. \& Li, Y. P. TGF-beta and BMP signaling in osteoblast, skeletal development, and bone formation, homeostasis and disease. Bone Res. 4, 16009 (2016).

14. Zaidi, S. K. et al. Integration of Runx and Smad regulatory signals at transcriptionally active subnuclear sites. Proc. Natl Acad. Sci. USA 99, 8048-8053 (2002).

15. Afzal, F. et al. Smad function and intranuclear targeting share a Runx2 motif required for osteogenic lineage induction and BMP2 responsive transcription. J. Cell. Physiol. 204, 63-72 (2005).

16. Lee, M. H., Kwon, T. G., Park, H. S., Wozney, J. M. \& Ryoo, H. M. BMP-2-induced Osterix expression is mediated by Dlx5 but is independent of Runx2. Biochem. Biophys. Res. Commun. 309, 689-694 (2003).

17. Rawadi, G., Vayssiere, B., Dunn, F., Baron, R. \& Roman-Roman, S. BMP-2 controls alkaline phosphatase expression and osteoblast mineralization by a Wnt autocrine loop. J. Bone Miner. Res. 18, 1842-1853 (2003).
18. Rodda, S. J. \& McMahon, A. P. Distinct roles for Hedgehog and canonical Wnt signaling in specification, differentiation and maintenance of osteoblast progenitors. Development 133, 3231-3244 (2006).

19. Salazar, V. S., Ohte, S., Capelo, L. P., Gamer, L. \& Rosen, V. Specification of osteoblast cell fate by canonical Wnt signaling requires Bmp2. Development 143, 4352-4367 (2016).

20. McBride, S. H. et al. Long bone structure and strength depend on BMP2 from osteoblasts and osteocytes, but not vascular endothelial cells. PLOS ONE 9, e96862 (2014).

21. McBride-Gagyi, S. H., McKenzie, J. A., Buettmann, E. G., Gardner, M. J. \& Silva, M. J. Bmp2 conditional knockout in osteoblasts and endothelial cells does not impair bone formation after injury or mechanical loading in adult mice. Bone 81, 533-543 (2015).

22. Feng, J. et al. Abnormalities in the enamel in bmp2-deficient mice. Cells Tissues Organs 194, 216-221 (2011).

23. Guo, F. et al. Bmp2 deletion causes an amelogenesis imperfecta phenotype via regulating enamel gene expression. J. Cell. Physiol. 230, 1871-1882 (2015).

24. Rakian, A. et al. Bone morphogenetic protein-2 gene controls tooth root development in coordination with formation of the periodontium. Int. J. Oral Sci. 5, 75-84 (2013).

25. Chen, T. \& Dent, S. Y. Chromatin modifiers and remodellers: regulators of cellular differentiation. Nat. Rev. Genet. 15, 93-106 (2014).

26. Heuston, E. F. et al. Establishment of regulatory elements during erythromegakaryopoiesis identifies hematopoietic lineage-commitment points. Epigenetics Chromatin 11, 22 (2018).

27. Jia, G. et al. Single cell RNA-seq and ATAC-seq analysis of cardiac progenitor cell transition states and lineage settlement. Nat. Commun. 9, 4877 (2018).

28. Huang, W., Yang, S., Shao, J. \& Li, Y. P. Signaling and transcriptional regulation in osteoblast commitment and differentiation. Front. Biosci. 12, 3068-3092 (2007).

29. Yaragatti, M., Basilico, C. \& Dailey, L. Identification of active transcriptional regulatory modules by the functional assay of DNA from nucleosome-free regions. Genome Res. 18, 930-938 (2008).

30. Ducy, P., Zhang, R., Geoffroy, V., Ridall, A. L. \& Karsenty, G. Osf2/Cbfa1: a transcriptional activator of osteoblast differentiation. Cell 89, 747-754 (1997).

31. Kim, Y. J., Lee, M. H., Wozney, J. M., Cho, J. Y. \& Ryoo, H. M. Bone morphogenetic protein-2-induced alkaline phosphatase expression is stimulated by Dlx5 and repressed by Msx2. J. Biol. Chem. 279, 50773-50780 (2004).

32. Maruyama, Z. et al. Runx2 determines bone maturity and turnover rate in postnatal bone development and is involved in bone loss in estrogen deficiency. Dev. Dyn. 236, 1876-1890 (2007).

33. Mevel, R., Draper, J. E., Lie, A. L. M., Kouskoff, V. \& Lacaud, G. RUNX transcription factors: orchestrators of development. Development 146, 17 (2019).

34. Arnold, M. A. et al. MEF2C transcription factor controls chondrocyte hypertrophy and bone development. Dev. Cell 12, 377-389 (2007).

35. Murakami, T. et al. Signalling mediated by the endoplasmic reticulum stress transducer OASIS is involved in bone formation. Nat. Cell Biol. 11, 1205-1211 (2009).

36. Liu, $\mathrm{H}$. et al. Analysis of zebrafish periderm enhancers facilitates identification of a regulatory variant near human KRT8/18. Elife 9, e51325 (2020).

37. Lee, S. U. \& Maeda, T. POK ZZBTB proteins: an emerging family of proteins that regulate lymphoid development and function. Immunol. Rev. 247, 107-119 (2012).

38. Michikami, I. et al. Kruppel-like factor 4 regulates membranous and endochondral ossification. Exp. Cell Res. 318, 311-325 (2012).

39. Kim, J. H. et al. Kruppel-like factor 4 attenuates osteoblast formation, function, and cross talk with osteoclasts. J. Cell Biol. 204, 1063-1074 (2014).

40. Tsuji, K et al. BMP2 activity, although dispensable for bone formation, is required for the initiation of fracture healing. Nat. Genet. 38, 1424-1429 (2006).

41. Chappuis, V. et al. Periosteal BMP2 activity drives bone graft healing. Bone 51, 800-809 (2012).

42. Takahashi, K. \& Yamanaka, S. Induction of pluripotent stem cells from mouse embryonic and adult fibroblast cultures by defined factors. Cell 126, 663-676 (2006).

43. Chen, X., Whitney, E. M., Gao, S. Y. \& Yang, V. W. Transcriptional profiling of Krüppel-like factor 4 reveals a function in cell cycle regulation and epithelial differentiation. J. Mol. Biol. 326, 665-677 (2003).

44. Birsoy, K., Chen, Z. \& Friedman, J. Transcriptional regulation of adipogenesis by KLF4. Cell Metab. 7, 339-347 (2008). 
45. Tao, $\mathrm{H}$. et al. Klf4 promotes dentinogenesis and odontoblastic differentiation via modulation of TGF- $\beta$ signaling pathway and interaction with histone acetylation. J. Bone Miner. Res. 34, 1502-1516 (2019).

46. Leboy, P. S., Beresford, J. N., Devlin, C. \& Owen, M. E. Dexamethasone induction of osteoblast mRNAs in rat marrow stromal cell cultures. J. Cell Physiol. 146, 370-378 (1991)

47. Ogata, Y. et al. Glucocorticoid Regulation of Bone Sialoprotein (BSP) gene expression. identification of a glucocorticoid response element in the bone sialoprotein gene promoter. Eur. J. Biochem. 230, 183-192 (1995).

48. Igarashi, M. et al. Inductive effects of dexamethasone on the gene expression of Cbfa1, Osterix and bone matrix proteins during differentiation of cultured primary rat osteoblasts. J. Mol. Histol. 35, 3-10 (2004).

49. Mikami, Y., Omoteyama, K, Kato, S. \& Takagi, M. Inductive effects of dexamethasone on the mineralization and the osteoblastic gene expressions in mature osteoblast-like ROS17/2.8 cells. Biochem. Biophys. Res. Commun. $\mathbf{3 6 2}$ 368-373 (2007)

50. Yang, W. et al. Bmp2 in osteoblasts of periosteum and trabecular bone links bone formation to vascularization and mesenchymal stem cells. J. Cell Sci. 126, 4085-4098 (2013).

51. Elefteriou, F. \& Yang, X. Genetic mouse models for bone studies-strengths and limitations. Bone 49, 1242-1254 (2011).

52. Ma, L. \& Martin, J. F. Generation of a Bmp2 conditional null allele. Genesis 42 , 203-206 (2005)

53. Katz, J. P. et al. Loss of Klf4 in mice causes altered proliferation and differentiation and precancerous changes in the adult stomach. Gastroenterology 128, 935-945 (2005).

54. Huang, W. \& Olsen, B. R. Skeletal defects in Osterix-Cre transgenic mice Transgenic Res. 24, 167-172 (2015). 